\title{
Die SIT als Praxis-Besonderheit
}

\author{
Weiterhin beklagen zahlreiche Kolleginnen und Kollegen Regress- \\ Drohungen durch regionale KVen aufgrund angeblich unwirtschaft- \\ licher Verordnungsweise in der Allergologie. Betroffene sollten sich \\ unbedingt wehren. Nachfolgend sind alle wesentlichen Argumente \\ dargestellt, die Sie in einem solchen Fall vorbringen können.
}

n der „Empfehlung zu Richtgrößen“ der KBV und der Spitzenverbände der Krankenkassen vom 10. Februar 1999 wird im Kapitel 2, Absatz 5, dargelegt, dass „bei der Bildung von Richtgrößen für Arzneimittel Verordnungen ausgenommen werden können, bei denen keine Anhaltspunkte

— für eine unwirtschaftliche Anwendung,

— für eine Verordnung außerhalb der zugelassenen Indikation oder

— für eine Mengenausweitung bestehen".

Verordnungen von Präparaten zur spezifischen Immuntherapie (SIT) durch allergologisch tätige Fachärzte entsprechen diesen Kriterien.

\section{Keine Anhaltspunkte für} unwirtschaftliche Anwendung

Die SIT ist laut WHO neben der Allergen-Karenz die einzige kausale Behandlung von allergischen Atemwegserkrankungen wie Rhinitis und Asthma. Damit bietet die SIT als einzige Therapieform Allergie-Patienten die Chance, ihre Krankheit dauerhaft zu heilen. Darüber hinaus wirkt die SIT im Sinne einer Allergie-Impfung präventiv, d.h. sie kann den so genannten Etagenwechsel und weitere Neusensibilisierungen verhindern [7]. Da keine andere Therapie diese Ziele erreicht, ist bei richtiger Indikationsstellung eine unwirtschaftliche Anwendung ausgeschlossen.

Gesundheitsökonomische Studien haben ein erhebliches Einsparpotenzial durch die SIT ermittelt $[2,4,5]$. So ist die jährliche Asthma-Inzidenz bei Allergikern unter einer rein symptomatischen Therapie 3-fach höher als bei einer SIT. Dies wird ebenfalls durch eine 2,5-fach höhere Remissionsrate von Asthma bestätigt [3].
Zur Wirtschaftlichkeit der Therapie trägt zudem bei, dass Packungsgrößen und Therapiedauer aufeinander abgestimmt sind, wodurch bei Einhaltung der klaren Anwendungsschemata keinerlei Medikamenten-Verluste entstehen.

Auch hat aktuell eine Facharztstudie die hohe Patienten-Compliance unter der SIT bestätigt [1]. Daher ergeben sich keine Anhaltspunkte für eine unwirtschaftliche Anwendung der SIT. Im Gegenteil: Eine strenge Richtgrößen-Regelung würde möglicherweise zum vorzeitigen Abbruch der SIT und damit zu einer unwirtschaftlichen Anwendung führen.

\section{Keine Anwendung außerhalb der zugelassenen Indikation}

Voraussetzung für eine SIT ist eine Diagnose durch den allergologisch erfahrenen Arzt, der neben einer ausführlichen Anamnese objektive Parameter wie Hauttest, Serum-IgE und gegebenenfalls Provokationstestungen zugrunde liegen. Einer SIT geht zumeist eine mehrjährige Krankengeschichte bzw. Beobachtungsphase voran. Daher sind Anwendungen außerhalb der Indikation wenig wahrscheinlich und bisher weder bekannt noch beschrieben.

Aufgrund der Allergie-spezifischen Wirkmechanismen der SIT wären diese auch wirkungslos. Die Indikation für eine SIT wird daher streng und nur bei entsprechender Compliance seitens des Patienten gestellt.

\section{Keine Anhaltspunkte für eine Mengenausweitung}

Die SIT ist eine aufwendige und zeitintensive Therapie, der sich ausschließlich Patienten mit entsprechendem Leidensdruck unterziehen. Trotz der Zunahme von Allergien sehen die Allergen-Her- steller in den letzten Jahren kein Mengenwachstum des SIT-Marktes beim Facharzt. Laut Verordnungsreport 1999 [6] betrug der Umsatz für SIT-Präparate im Bereich der GKV 187 Mio. DM.

Demnach stellt die SIT 0,5\% des GKV-Gesamtmarktes für Fertigarzneimittel dar. Für Broncholytika/Antiasthmatika gibt der Verordnungsreport dagegen 10-fach höhere Ausgaben von 1.873 Mio. DM an. Ca. 80\% der SITPräparate werden in Deutschland von den Facharztgruppen der Dermatologen oder HNO-Ärzte verordnet. Insgesamt verordnet die Fachgruppe der Dermatologen lediglich $2,1 \%$, die der HNOÄrzte 1,1\% des GKV-Arzneimittelmarktes. Selbst wenn aufgrund zunehmender Morbidität eine Zunahme der indikationsgerecht eingesetzten SIT erfolgte, wären die kurzfristigen Auswirkungen auf die Gesamt-Arzneimittelkosten äußerst gering. Langfristig führt die SIT zu Einsparungen für das Gesundheitswesen in Millionenhöhe. Der Verwaltungsaufwand zur Beurteilung von individuellen Praxisbesonderheiten stünde in keinem angemessenen Verhältnis.

Die eingangs genannte „Empfehlung zu Richtgrößen“ führt SIT-Präparate zur Behandlung der InsektengiftAllergie bereits in Anlage 2 auf. Insgesamt würde die Aufnahme der SITPräparate als Facharzt-Verordnung in der Anlage 3 die adäquate Versorgung von Allergie-Patienten sichern.

Die KV-Brandenburg und die Verbände der Krankenkassen haben in ihrer Vereinbarung vom 23. September 1999 SIT-Präparate bei allergischen Erkrankungen in Anlage 3 als Fall-bezogene indikationsabhängige Praxis-Besonderheit gewertet. Die KBV und weitere KVen haben eine ähnliche Einschätzung gegenüber dem ehemaligen ÄDA-Vorsitzenden, Herrn Dr. Josef Wenning, geäußert.

Aufgrund dieser Sachlage sollte auch in anderen KV-Bezirken dem Beispiel Brandenburgs gefolgt und die SIT bei fachärztlicher Verordnung als PraxisBesonderheit anerkannt werden, um damit die Versorgung von AllergiePatienten in allen KV-Bereichen zu sichern. Prof. Dr. L. Klimek, Wiesbaden

\section{Literatur beim Verfasser}

